\title{
Robotic Pancreaticoduodenectomy: Technical Considerations
}

\author{
$\operatorname{Marco~Marino~}^{1} \cdot \operatorname{Gaspare}_{\text {Gulotta }}{ }^{1}$ - Andrzej L. Komorowski ${ }^{2}$
}

Received: 9 December 2016 / Accepted: 13 March 2017 / Published online: 20 March 2017

(C) Association of Surgeons of India 2017

\begin{abstract}
Robotic surgery can help to overcome some technical limitations of laparoscopic pancreaticoduodenectomy thanks to EndoWrist instrumentations and the 3D view. Despite the potential benefits, its employment is still low and controversial. We focused on some important technical details crucial for a safe robotic pancreatectomy. After performing 52 robotic pancreatic resections that included 10 pancreatoduodenectomies, the authors describe their technique. The review of literature on robotic and laparoscopic duodenopancreatectomy is also performed in order to evaluate possible benefits of the robotic platform. We describe the step-by-step surgical procedure, analyzing all possible troubleshooting occurring in an initial center experience. The estimated blood loss as well as the length of stay was reduced by the robotic approach. We did not observe any significant increase of pancreatic fistula rate and all other postoperative complications despite our initial learning curve. Robotic pancreatoduodenectomy is a technically advanced procedure that requires important laparoscopic and robotic skills but it shows to be safe, feasible with some clear advantages in the bleeding control and in the reconstructive phase of the procedure.
\end{abstract}

Keywords Robotic pancreatoduodenectomy · Operative technique $\cdot$ Pancreatic fistula $\cdot$ Learning curve

Marco Marino

marco.vito.marino@gmail.com

1 Department of Emergency and General Surgery, P. Giaccone Hospital, University of Palermo. Giuffrè L. str. 5, 90127 Palermo, Italy

2 Department of Surgical Oncology, Maria Skłodowska-Curie Memorial Institute of Oncology Cancer Centre, Garncarska str. 11, 31-115 Kraków, Poland

\section{Introduction}

The laparoscopic surgery is a safe and feasible surgical approach to distal pancreatectomy [1]. It shows clear short-term benefits increasing the spleen preservation rate. It also seems to be associated with promising oncologic results [1, 2]. However, its use is still controversial in more challenging pancreatic procedures like pancreaticoduodenectomy (PD), central pancreatectomy, or when a vascular resection is deemed necessary.

Even if the first description of robotic pancreatectomy was reported already in 1994 by Gagner and Pomp [3], the widespread application of the laparoscopic PD is still limited due to some technical issues present not only during the lymph node dissection around the major vascular structures but also during the reconstructive phase and in cases when vascular control is required.

So it is not a big surprise that only 341 laparoscopic PDs were reported in literature until 2011, while between 2012 and 2015 after the wide acceptance of the robotic platform, the total number of laparoscopic and robotic PD's reported increased more than twofold (725) [4].

The presence of surgical dexterity associated with 7 degrees of movements offered by the robotic instruments and the absence of physiological tremor thanks to a steady surgical 3D camera platform allow surgeon to perform each step of the procedure in a more comfortable position helping him in some crucial surgical phases of the operation [5].

In the present paper, we present our surgical technique of robotic-assisted laparoscopic pylorus-preserving PD with a Longmire-Traverso reconstruction that until now is not a standardized technique, in which the da Vinci Si surgical system (Intuitive Surgical, Sunnyvale, California, USA) is employed for the entire operation. We also discuss its technical aspects, giving some technical tricks and personal considerations. Results of robotic PD compared to those of laparoscopic and open approach are also discussed evaluating the possible benefits of robotic surgery. 


\section{Technique Description}

Robotic pancreatic surgery program started in our University Hospital in Palermo in 2012. Until July 2016, there have been 52 pancreatic resections performed, including 10 PDs ( 8 for pancreatic ductal adenocarcinoma and 2 for pancreatic neuroendocrine tumors).

In case of a pancreatic malignancy, all patients with small $(<3 \mathrm{~cm})$ tumors without evidence of lymph node metastases or vascular involvement at the preoperative work-up were considered eligible for robotic PD.

Patients with known locally unresectable disease on preoperative imaging underwent neoadjuvant therapy, followed by restaging and operative resection. The standard adjuvant therapy at our institution involved 2 months of gemcitabine before and after radiation (50-40 cGy) plus 5-fluorouracile and folinic acid; chemotherapy (CHT) is generally started within 8 weeks of surgery.

The patient under general endotracheal anesthesia is placed in supine position with legs apart in a reverse Trendelenburg's (Fowler's) position, slightly tilted to the left.

The patient's console comes from the head of the patient; the console of surgeon is in the corner of the operating room. The scrub-nurse stays on the left of the patient, while the bed assistant is between the patient's legs.

We use the following five ports (Fig. 1):

1. 12-mm optic port located in the right pararectal area

2. $8-\mathrm{mm}$ robotic trocar for arm number 1 placed in the left flank in mid-clavicular line (R1),

3. $8-\mathrm{mm}$ port placed in the right flank for arm number 2 (R2)

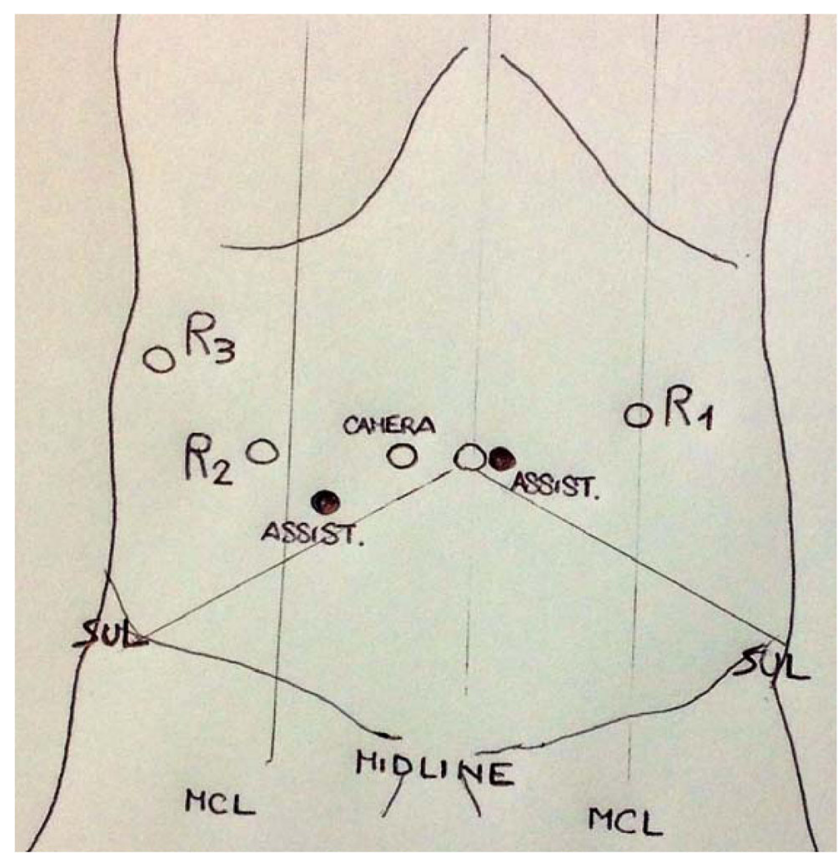

Fig. 1 Trocar/port placement
4. $8-\mathrm{mm}$ port located in the right hypochondriac region for the arm number 3 (R3)

5. One or two 12-mm assistant ports located one in the umbilical area, and the second in right flank medially to arm number 2 .

This lay-out has been previously described by Prof. Giulianotti [6], and allows to perform the Kocher maneuver of the duodenum using the third stable retractor arm coming from the right and lifting up the duodenum.

Other techniques describe a different position of the arm number 3 or a dynamic position of the trocars.

The operation starts with the opening of the gastrocolic ligament and the hepatic flexure of the colon is taken down, the gastroepiploic vessels are exposed up to its origin, the duodenum is exposed, and the superior mesenteric vein is identified. After the evaluation of resectability, we perform a full Kocher maneuver that must be more extended than in open approach until we reach the left lateral border of the aorta (Fig. 2). In this step, it is necessary to have a clear understanding of the anatomy before the beginning of the demolitive part, evaluating all anatomic landmarks like the Henle's trunk that must be sectioned to achieve the superior mesenteric vein. We do not perform an extensive inter-aortocaval lymphoadenectomy that we carry on only in cases of macroscopic involvement.

After the division of the gastroepiploic vessels, the duodenum is transected using an EndoGia linear 45-mm stapler.

An expert assistant can perform a good traction and help the operator to maintain a clean operative field. We continue with the dissection of the hepatoduodenal ligament and the gallbladder is taken down, and the common bile duct is dissected and isolated. For this step, we use a monopolar hook, Harmonic or vessel sealer in cases of fatty tissue, and when the control of hemostasis is troublesome.

We identified the common hepatic artery and we isolate and follow it in order to identify the gastroduodenal artery that we control by hand-sewn ligation (Fig. 3).

The robot-sewn ligation of the artery is one of the key points of the procedure. It gives us a more precise control of

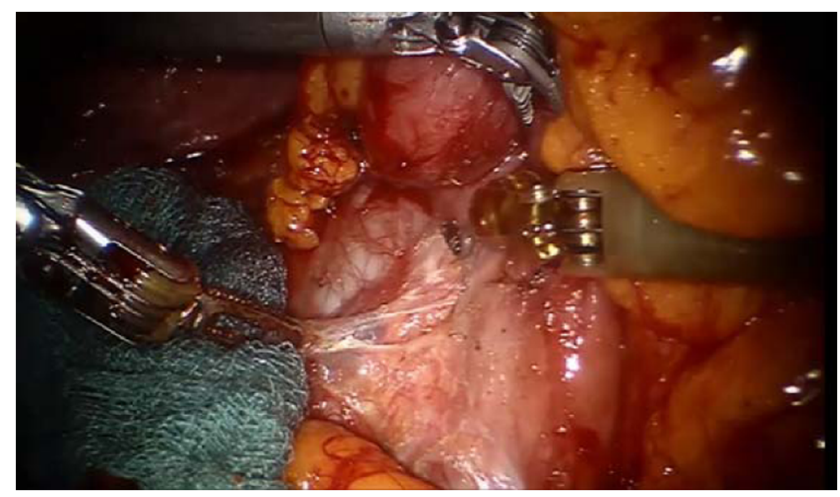

Fig. 2 Full Kocher maneuver 


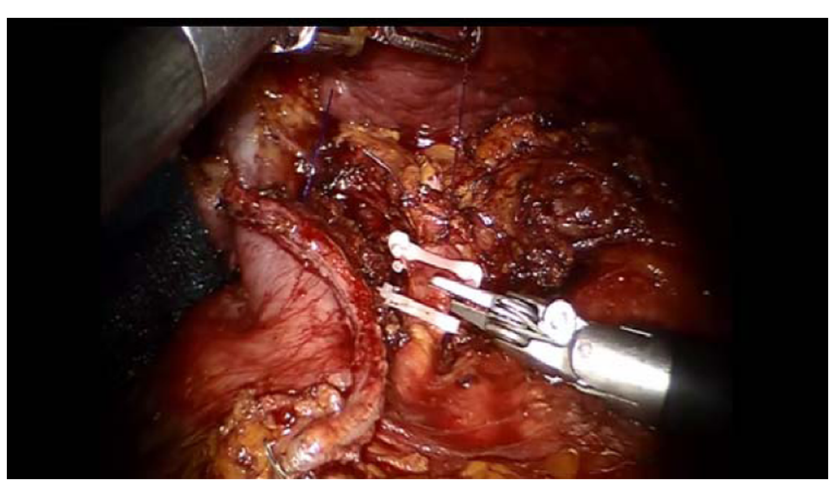

Fig. 3 Identification and isolation of the common hepatic artery, identification, and control of gastroduodenal artery

this vessel than Hem-o-Lock. Afterwards, the common bile duct can be transected and sent for a pathology examination and closed by a bulldog clamp inserted through robotic Prograsp. It is mandatory to have a clear idea of vascular anatomy before starting the procedure, so we generally perform a $3 \mathrm{D}$ radiological vascular reconstruction before surgery.

The pancreas is dissected in its superior and posterior margin in order to create a retropancreatic tunnel. We generally do not do many efforts to encircle the pancreatic parenchyma because we do not believe it is necessary.

We continue with the demolitive part performing stay sutures at the inferior and superior border of the pancreas and the transection is then carried out using ultrasonic shears to incise the first centimeter of the pancreatic neck and then using a bipolar scissors in order to visualize the Wirsung's duct (Fig. 4). An internal drain is inserted even if it seems that it does not reduce the postoperative pancreatic fistula rate. The proximal part of pancreatic remnant is sent for pathology examination. The first jejunal loop is transected using a $60-\mathrm{mm}$ laparoscopic stapler and mobilized from the Treitz's ligament to the root of the transverse mesocolon. After that, we pull it to the right of the superior mesenteric artery axis.

We perform this submescolic part of the procedure lifting up the transverse colon with the third arm and connecting the transected jejunal loop with a silicon drain in order to help the

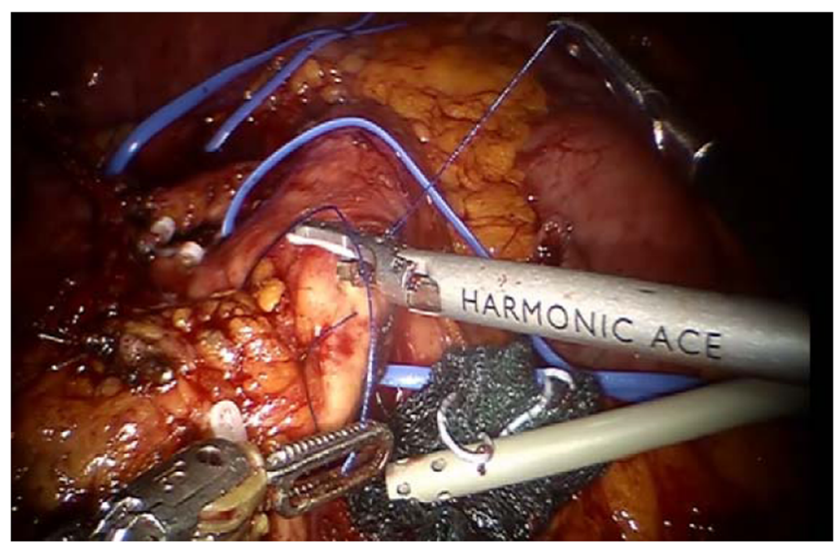

Fig. 4 Process of Wirsung's duct visualization transposition to a supramesocolic area. Some surgeons perform this step directly from the right axis of SMA performing a massive traction to the bowel loop and performing immediately an extensive Kocher maneuver.

The last demolitive part is completed with the uncinate process dissection from the distal to proximal part of the superior mesenteric vein (SMV) with a good control of each jejunal branch. This last part is challenging in laparoscopy as well as in open surgery for the presence of many arterial and venous branches, surrounded by fat tissue and nerves. The magnified view of robotic console can help surgeons to overcome these difficulties allowing for selective control of the vessels using both monopolar or bipolar energy and obtaining a good oncologic adequacy.

The lymph node dissection, the uncinate process dissection, and the liver hilum dissection are crucial steps of the procedure and are relatively easy to perform by robotic approach.

The reconstructive phase starts with a pancreatojejunostomy with an external row of polydioxanone suture (PDS) 4.0 interrupted and a Wirsung-mucosa anastomosis using a Prolene 5.0 in traditional fashion (Fig. 5).

Afterwards, we continue performing a robot-sewn biliodigestive anastomosis, performing a running suture posteriorly and interrupted suture anteriorly using PDS 5.0 and at the end, an antecolic gastrojejunostomy using the same single jejunum loop with a running PDS 3.0.

Once the anastomosis is completed and an accurate hemostasis achieved, we insert three drains: the first one behind the pancreaticojejuno anastomosis, the second one over pancreaticojejuno anastomosis, and the last one below the liver.

The specimen is extracted through a Pfannenstiel's incision.

\section{Discussion}

Minimally invasive techniques of pancreatic resection have had a difficult start. Laparoscopy approach, although seen as

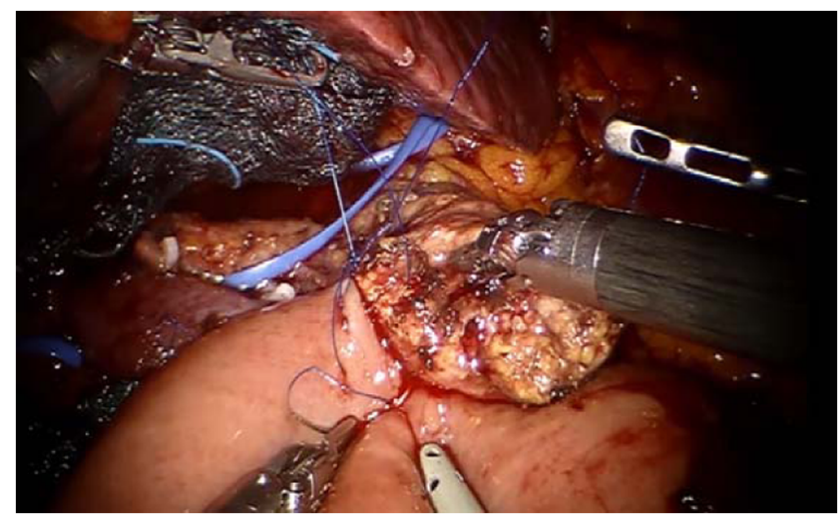

Fig. 5 Reconstructive phase of procedure 
an interesting or even preferred option for distal pancreatectomy, was considered for a long time not suitable for pancreatoduodenectomy, because of its extreme level of technical difficulty. However, with the advent of robotic surgery, several limitations of the laparoscopy approach seemed possible to overcome and thus the minimally invasive resections of the pancreas saw a new boost of interest.

One of the most important factors that influenced the widespread of robotic PD is the faster learning curve ( 80 cases) and the possibility to use a double console to involve novice surgeons in large part of operations as showed by Boone. The literature data show a decreased $(9.1 \%)$ conversion rate with robot compared to that of laparoscopic surgery and only 20 cases are necessary to reduce this rate from 35 to $3.3 \%$ [7].

In our experience of 10 reported robotic $\mathrm{PD}$, we have had only one conversion to open surgery caused by the tumor adherence to the SMV and portal vein not diagnosed preoperatively. At the same time, we believe that in future, the robotic platform can also be employed to perform vascular resection in case of portal vein lateral attachment [8].

The median operative time reported in our experience (517 $\mathrm{min}$ ) seems to be less than that for laparoscopy (624 $\mathrm{min}$ ) but more than that for open (423 $\mathrm{min}$ ) as confirmed by other series [9], but of course, we must consider the learning curve and specific patient-related factors. What we observed during the operation is a different perception of the operative field, with a magnified and more accurate vision that can justify a higher operative time in comparison to that of laparoscopic approach where the length of operation is generally caused by a difficult and complex dissection.

Several studies $[10,11]$ showed that blood loss is significantly less in robotic surgery than that in open approach, and comparing the transfused blood units, patients who undergo open procedures require more blood units to be transfused than those who undergo the minimally invasive approach. We can confirm this observation, because not only we saw a decreased blood loss in comparison to that of open approach but also to laparoscopic surgery (70 vs. 450 vs. $500 \mathrm{ml}$, respectively). The possibility of switching among monopolar and bipolar energy allows to have an easier bleeding control.

The analysis of literature shows that the rate of bile leak, delayed gastric emptying, and pancreatic fistula after robotic PD surgery is similar to that of open approach (17 vs. 10 $16 \%$ ) [12-15] and fortunately, half of these are not clinically significant (grade A fistula, which can be managed by drainage alone), but what is really important to underline is the "heightened" awareness of surgeons in detecting leaks due to less postoperative adhesions.

In a recent systematic review, there was no significant difference in postoperative pancreatic fistula incidence and mortality between robotic and open approaches but a trend towards improvement in clinically significant fistula rates with surgeon experience was evident [9].
It can be difficult to define the real clinical impact of several factors influencing fistula occurrence since studies on the topic are biased, the number of soft glands with smaller ducts is not taken into account, and the impact of learning curve is frequently neglected. However, Polanco et al. [16] reported four risk factors for postoperative pancreatic fistula after robotic PD: duct size $<4 \mathrm{~mm}$, estimated blood loss, small tumor size, and BMI. In line with these findings, in our experience, we had one pancreatic fistula in an obese patient with a 3-mm Wirsung's duct.

Considering the small number of patients who underwent a robotic $\mathrm{PD}$, our data regarding morbidity and mortality rate are clearly insufficient, but we did not have until now any case of 30-day as well as 90-day mortality.

In an interesting analysis of 250 consecutive robotic pancreatic resections, an overall 30-day mortality of $0.8 \%$ and a 90-day mortality of $3.8 \%$ have been shown. The morbidity still remains considerable and is comparable to open surgery (grades 3 and 4 Clavien complications 10 and $11 \%$, respectively) even if it declines with experience [17].

The data about the oncologic outcome in the literature are not clear, not always collected in a standardized manner regarding the margin status and patient selection criteria for mini invasive surgery. Further evaluation of this aspect of robotic pancreatic surgery is definitely needed.

The median number of harvested lymph nodes in our group is 25 , similar to 29 reported in the literature and comparable to open approach. The oncologic quality parameters like $\mathrm{R} 0$ and $\mathrm{R} 1$ resection margins that we have obtained $(9 / 10$ and $1 / 10$, respectively) confirm that robotic surgery is oncologically equivalent to open surgery as confirmed by the results of an Asian group (78.9-100\% vs. 68.3-87.5\%) [18].

The comparison of oncologic outcomes of open and robotic approach in our experience will be the subject of future research. Until now, an interesting study showed not only a shorter hospitality stay in the robotic group but also a shorter delay ( 27 vs. $41 \%$ ) in starting adjuvant treatments caused by poor functional status of patients after surgery [19].

An improved progression-free survival (but not overall survival) was observed in the robotic group patients. This may be a reflection of the differences in the delay of the start of adjuvant treatment. The lower impact of robotic surgery on immunological response and humoral balance can lead to a better and faster recovery after surgery and thus cause a shorter delay of treatment with a reduced waiting time before chemotherapy [20].

In a recent study performed by the European Study Group for Pancreatic Cancer (ESPAC-3), the completion of all six cycles of adjuvant chemotherapy was an independent predictive prognostic factor of overall survival whereas time to starting adjuvant chemotherapy was not prognostic if chemotherapy was started within 12 weeks after surgery [21].

The most important therapeutic advantage is seen in patients who complete all six cycles of $\mathrm{CHT}$ and receive adjuvant treatment in time (median survival 28.0 vs. 14.6 months). In fact, there 
is no survival disadvantage from delaying the start of CHT for up to 12 weeks after surgery and no survival advantage for starting early treatment, within 8 weeks of surgery ( 22.6 vs. 24.2 months), so what is really important is to treat fully recovered patients. The time to start of therapy was only identified as an important factor for the subgroup of patients who did not complete therapy with reduced survival observed in patients starting chemotherapy early [21].

In conclusion, in many ways, the future of robotic pancreatic surgery seems optimistic. The main obstacle for this technique remains the costs (studies analyzing the economic sustainability are required), the length of operative time, the lack of haptic feedback, and the necessity of another skilled robotic assistant at the table [22]. But the technique seems safe and relatively easy at least when compared with laparoscopic surgery. In our experience, the main advantages of the robot can be mostly appreciated during the uncinate process dissection, the lymphadenectomy, and during reconstruction.

Regarding the training, the use of double console can give the novice surgeons the opportunity to perform some steps of the procedure under the guidance of the experienced operator, and it is always important to remind that a good team work is a key to success. The impact of the new Xi robotic platform and possibilities it offers still need more studies.

\section{Compliance with Ethical Standards}

Conflict of Interest The authors declare that they have no conflict of interest.

\section{References}

1. Mehrabi A, Hafezi M, Arvin J, Esmaeliziadeh M, Garoussi C, Emami G, Kossler-Ebs J, Muller-Stich B, Buchler M, Hackert T, Diener MK (2015) A systematic review and meta-analysis of laparoscopic versus open distal pancreatectomy for benign and malignant lesions of the pancreas: it's time to randomize. Surgery 157(1): 45-55

2. Kang CM, Kim DH, Lee WJ, Chi HS (2011) Conventional laparoscopic and robot-assisted spleen-preserving pancreatectomy: does da Vinci have clinical advantages? Surg Endosc 25(6):2004-2009

3. Gagner M, Pomp A (1994) Laparoscopic pylorus-preserving pancreatoduodenectomy. Surg Endosc 8(5):408-410

4. Boggi U, Palladino S, Massimetti G, Vistoli F, Caniglia F, De Lio N, Perrone V, Barbarello L, Belluomini M, Signori S, Amorese G, Mosca F (2015a) Laparoscopic robot-assisted versus open total pancreatectomy: a case-matched study. Surg Endosc 29(6):14251432

5. Boggi U, Amorese G, Vistoli F, Caniglia F, De Lio N, Perrone V, Barbarello L, Belluomini M, Signori S, Mosca F (2015b) Laparoscopic pancreaticoduodenectomy: a systematic literature review. Surg Endosc 29(1):9-23

6. Giulianotti PC, Sbrana F, Bianco FM et al (2010) Robot-assisted laparoscopic pancreatic surgery: single-surgeon experience. Surg Endosc 24(7):1646-1657

7. Boone B, Zenati M, Hogg M, Steve J, Moser A, Bartlett D, Zeh H, Zureikat A (2015) Assesment of quality outcomes for robotic pancreaticoduodenectomy: identification of the learning curve. JAMA Surg 150(5):416-422

8. Wright GP, Zureikat AH (2016) Development of minimally invasive pancreatic surgery: an evidence-based systematic review of laparoscopic versus robotic approaches. J Gastrointest Surg 20(9): $1658-1665$

9. Zhang J, Wu WM, You L, Zhao YP (2013) Robotic versus open pancreatectomy: a systematic review and meta-analysis. Ann Surg Oncol 20(6):1774-1780

10. Buchs NC, Addeo P, Bianco FM et al (2011) Robotic versus open pancreatoduodenecotmy: a comparative study at a single institution. World J Surg 35(12):2739.2746

11. Chalikonda S, Aguilar-Saavedra JR, Walsh RM (2012) Laparoscopic robotic-assisted pancreatoduodenectomy: a casematched comparison with open resection. Surg Endosc 26(9): 2397-2402

12. Hackert T, Werner J, Büchler MW (2011) Postoperative pancreatic fistula. Surgeon 9(4):211-217

13. Asbun HJ, Stauffer JA (2012) Laparoscopic vs open pancreaticoduodenectomy: overall outcomes and severity of complications using the Accordion Severity Grading System. J Am Coll Surg 215(6):810-819

14. Addeo P, Delpero JR, Paye F, Oussoultzoglou E, Fuchshuber PR, Sauvanet A, Sa Cunha A, Le Treut YP, Adham M, Mabrut JY, Chiche L, Bachellier P (2014) Pancreatic fistula after a pancreatoduodenectomy for ductal adenocarcinoma and its association with morbidity: a multicentre study of the French Surgical Association. HPB (Oxford) 16(1):46-55

15. Coratti A, Di Marino M, Coratti F, Baldoni G, Guerra F, Amore Bonapasta S, Bencini L, Farsi L, Annecchiarico M (2016) Initial experience with robotic pancreatic surgery: technical feasibility and oncological implications. Surg Laparosc Endosc Percutan Tech 26(1):31-37

16. Polanco P, Zenati S, Hogg M, Shakir M, Boone B, Bartlett D, Zeh $\mathrm{H}$, Zureikat A (2016) An analysis of risk factors for pancreatic fistula after robotic pancreaticoduodenectomy:outcomes from a consecutive series of standardized pancreatic reconstruction. Surg Endosc 30(4):1523-1529

17. Zureikat AH, Moser AJ, Boone BA, Bartlett DL, Zenati M, Zeh HJ (2014) 3rd. 250 robotic pancreatic resections: safety and feasibility. Ann Surg. 2013;258(4):554-9; discussion 559-62. Erratum in: Ann Surg.; 260(3):566.

18. Lai EC, Yang GP, Tang CN (2012) Robot-assisted laparoscopic pancreatoduodenectomy versus open pancreatoduodenectomy- $\mathrm{a}$ comparative study. Int J Surg 10(9):475-479

19. Bernard AC, Davenport DL, Chang PK, Vaughan TB, Zwischenberger JB (2009) Intraoperative transfusion of $1 \mathrm{U}$ to 2 $\mathrm{U}$ packed red blood cells is associated with increased 30-day mortality, surgical-site infection, pneumonia, and sepsis in general surgery patients. J Am Coll Surg 208(5):931-937

20. Croome KP, Farnell MB, Que FG, Reid-Lombardo KM, Truty MJ, Nagorney DM, Kendrick ML (2014) Total laparoscopic pancreaticoduodenectomy for pancreatic ductal adenocarcinoma: oncologic advantages over open approaches? Ann Surg 260(4): 633-638

21. Valle J, Palmer D, Jackson R, Cox T, Neoptolemos J, Ghanch P, Rawcliffe C, Bassi C, Stocken D, Cunnigham D, O'Reilly D, Goldstein D, Robinson B, Karapetis C, Scarfe A, Lacaine F, Sand J, Izbicki J, Mayerle J, Dervenis C, Olah A, Butturini G, Lind P, Middleton M, Anthoney A, Sumpter K, Carter R, Buchler M (2014) Optimal duration and timing of adjuvant chemotherapy after definitive surgery for ductal adenocarcinoma of the pancreas: ongoing lessons from the ESPAC-3 study. J Clin Oncol 32(6):504-512

22. Nappo G, Perinel J, El Bechwaty M, Adham M (2016) Minimally invasive pancreatic resection: is it really the future? Dig Surg 33(4): 284-289 